\begin{tabular}{|c|l|}
\hline Title & Transmission and reflection times of phonon packets propagating through superlattices \\
\hline Author(s) & Mizuno, Seiji; Tamura, Shin-ichiro \\
\hline Citation & $\begin{array}{l}\text { Physical Review B, 50(11), 7708.7718 } \\
\text { https://doi.org/10.1103/PhysRevB.50.7708 }\end{array}$ \\
\hline Issue Date & 199409 \\
\hline Doc URL & http://hdl.handle.net/2115/51001 \\
\hline Rights & ○ 1994 The A merican Physical Society \\
\hline Type & article \\
\hline File Information & Phys. Rev. B 50 7708.pdf \\
\hline
\end{tabular}

Instructions for use 


\title{
Transmission and reflection times of phonon packets propagating through superlattices
}

\author{
Seiji Mizuno and Shin-ichiro Tamura \\ Department of Engineering Science, Hokkaido University, Sapporo 060, Japan
}

(Received 6 May 1994)

\begin{abstract}
We study theoretically the asymptotic phase times of phonon packets scattered off the singleand double-superlattice structures which act as opaque barriers for phonons analogous to potential barriers for electrons. The analytical expressions for the transmission and reflection times are derived. For the systems consisting of semiconductor superlattices of the size of $1000 \AA$ we find that the time advance or delay of the transmitted and reflected packets range from 10 ps for a single-superlattice structure to $1 \mathrm{~ns}$ at resonances for a double-superlattice structure. These effects should be observable in the phonon generation and detection experiments utilizing a picosecond laser technique.
\end{abstract}

\section{INTRODUCTION}

Synthetic semiconductor heterostructures or superlattices are useful not only for the application to electronic devices of nanometer dimensions but also for the design of various phonon optics devices such as the phonon mirror, the phonon reflector, the phonon resonator, and so on. ${ }^{1,2}$ Stimulated by the idea for fabricating the latter devices, we have proposed in previous papers ${ }^{3,4}$ systems for phonons based on multiple superlattice structures. The key idea is to utilize the fact that in periodic superlattices Bragg reflections occur for long wavelength phonons, or the superlattices act as opaque barriers for phonons within the frequency gaps induced by the periodicity much longer than the lattice spacing. If a periodicity of a superlattice is $30 \AA$ or longer, the lowest frequency gap of phonons is produced in the sub- $\mathrm{THz}$ range. The wavelength of $1-\mathrm{THz}$ phonons is typically $30-50 \AA$ and their mean free path in pure solids is about $1 \mathrm{~mm}$ or longer at low temperatures. Therefore, these highfrequency phonons propagate ballistically through the systems with linear dimensions in the nanometer range.

In the present work, we study the dynamical aspect of phonons propagating in superlattices, which has not been discussed before. On the analogy with electron systems, the ballistic phonons propagating through a superlattice barrier can be regarded as a tunneling if their frequencies within a frequency gap are concerned. In the past two decades, extensive studies ${ }^{5,6}$ have been conducted to understand quantum transport properties related to the electron tunneling through the single and multiple potential barriers. Recently, the time for the completion of a tunneling through the single- and double-barrier structures has attracted much attention ${ }^{7,8}$ but the situation remains still controversial.

One of the useful concepts related to the tunneling is the asymptotic phase times defined by the energy derivatives of the scattering phase shifts. The phase times are believed to be relevant to describe the motion of wave packets narrow in the momentum space. There are several other times such as dwell time, i.e., the mean time spent by an incident particle in the barrier region, traversal time, and so on, which are proposed to describe the temporal aspect of the tunneling. However, there exits no direct comparison of these theoretical tunneling times with experiments. This is partly because the characteristic time scale for electrons to tunnel through a potential barrier of $1 \mathrm{~nm}$ thickness is typically of the order of femtoseconds, which is shorter than the measurable time by commonly available methods. The tunneling occurs as a result of the wave nature of electrons. Thus, it should also occur for classical waves, such as, light and sound waves, and their quanta, i.e., photons and phonons.

Recently, we have studied by numerical simulations the time evolutions of phonon packets incident on the single and double-barrier structures for phonons realized by semiconductor superlattices. ${ }^{9}$ In the asymptotic regions far from the barriers both the time delay and advance are observed for the transmitted and reflected packets. To explain these results of the simulations, we develop in the present paper, the analytical calculations for the transmission and reflection times associated with the interaction of phonons with one-dimensional elastic barriers composed of periodic superlattices. The exact and useful approximate expressions for the phase times which well describe the delay times for phonon packets narrow in the wave-vector space are derived. Because phonons travel at sound velocities much slower than the velocities of electrons and also, as described above, their mean free path is macroscopic in pure samples at sub- $\mathrm{THz}$ frequencies, it is expected that the measurement of the time delay or advance of phonon packets scattered off the elastic barriers is much easier than the case of electrons.

In Sec. II, we formulate the transmission and reflection times of phonons propagating through a singlebarrier system. In Sec. III, we examine the phase times of phonons in a symmetric double-barrier structure, in which the resonant transmission occurs. For both systems, numerical examples assuming Gaussian phonon packets and GaAs/AlAs superlattice systems are illustrated . Also, the shapes of the transmitted and reflected packets are studied for the case of a double-barrier structure. In Sec. IV, a summary and conclusions are given. 


\section{SINGLE-BARRIER SYSTEM FOR PHONONS}

In this section, we consider a phonon packet incident on a single-barrier system for phonons realized by a periodic superlattice. The system consists of an alternate stacking of different elastic layers $A$ and $B$ sandwiched between other materials $X$ and $Y$, i.e., $X(A B)^{N} Y$, where $X$ is the substrate of the superlattice, $Y$ denotes the substance where the detection of the transmitted phonon packet is made, and $N$ is the number of unit periods $A B$. The transmission and reflection rates of monochromatic phonons through the periodic superlattice with a finite number of periodicities have been derived analytically in a previous paper. ${ }^{3}$ In Sec. II A, we briefly summarize the main results of Ref. 3 and in Sec. IIB, we define the asymptotic forms of the time dependent phonon packets scattered off the elastic barrier as the superpositions of the phonon fields of various wave numbers. In Sec. II C, we introduce the asymptotic phase times for the transmitted and reflected phonon packets which describe the times needed for the completion of the transmission and reflection. In Sec. IID, we derive the approximate expressions for the phase times to clarify the origin of these time advance and delay. Finally, in Sec. II E, numerical examples for the time advance (or delay) are given for a Gaussian phonon packet.

\section{A. Stationary scattering problem}

We study the case where the wave vector of phonons is perpendicular to the interfaces of the layering structure and three phonon modes are decoupled from each other, i.e., we treat only one mode, e.g., the longitudinal mode. Also, the continuum model which should be valid for sub$\mathrm{THz}$ phonons is assumed.

We formulate the transmission or reflection of phonons in a superlattice as a stationary scattering problem in one dimension. Suppose the case where the displacement field of incident phonons in the substrate is represented by the plane wave $e^{i k x} \quad\left(k=k_{X}\right)$ of unit amplitude. The incident phonons are scattered from the interfaces between dissimilar layers constituting the superlattice. The displacement fields of the transmitted and reflected phonons in the substrate and the detector layer are expressed as $t(k) e^{i k x}$ and $r(k) e^{-i k x}$, respectively. Here, $t(k)$ and $r(k)$ are the transmission and reflection amplitudes, respectively, and we assume that the substrate and the detector layer consist of the same material, i.e., $X=Y$.

For a superlattice with $N$ periodicities, the transmission and reflection amplitudes are calculated analytically. ${ }^{3}$ The expressions of $t$ and $r$ for phonons of frequency $\omega$ are given by

$$
\begin{aligned}
& t=\frac{2 i e^{-i k N D}}{\left[\left(Z_{X} / Z_{A}\right) \sigma-\left(Z_{A} / Z_{X}\right) \zeta\right] S(N)+2 i C(N)} \\
& r=\frac{\left[\left(Z_{X} / Z_{A}\right) \sigma+\left(Z_{A} / Z_{X}\right) \zeta-i(\lambda-\mu)\right] S(N)}{\left[\left(Z_{X} / Z_{A}\right) \sigma-\left(Z_{A} / Z_{X}\right) \zeta\right] S(N)+2 i C(N)}
\end{aligned}
$$

where

$$
\begin{aligned}
& S(N)=\left(\frac{\mu+\lambda}{|\mu+\lambda|}\right)^{N+1} \frac{\sinh N \theta}{\sinh \theta} \\
& C(N)=\left(\frac{\mu+\lambda}{|\mu+\lambda|}\right)^{N} \cosh N \theta \\
& \cosh \theta \equiv\left|\frac{\mu+\lambda}{2}\right|
\end{aligned}
$$

and

$$
\begin{aligned}
& \lambda=\cos k_{A} d_{A} \cos k_{B} d_{B}-\frac{Z_{A}}{Z_{B}} \sin k_{A} d_{A} \sin k_{B} d_{B} \\
& \sigma=\sin k_{A} d_{A} \cos k_{B} d_{B}+\frac{Z_{A}}{Z_{B}} \cos k_{A} d_{A} \sin k_{B} d_{B} \\
& \zeta=-\sin k_{A} d_{A} \cos k_{B} d_{B}-\frac{Z_{B}}{Z_{A}} \cos k_{A} d_{A} \sin k_{B} d_{B} \\
& \mu=\cos k_{A} d_{A} \cos k_{B} d_{B}-\frac{Z_{B}}{Z_{A}} \sin k_{A} d_{A} \sin k_{B} d_{B}
\end{aligned}
$$

In these equations, $d_{A}$ and $d_{B}$ are the thicknesses of $A$ and $B$ layers, respectively; $D\left(=d_{A}+d_{B}\right)$ is the unit period of the superlattice; $Z_{i}=\rho_{i} v_{i}(i=A, B$, and $X)$ is the acoustic impedance given by the product of the mass density $\rho_{i}$ and the sound velocity $v_{i} ; k_{i}=\omega / v_{i}$ is the wave number. The above expressions (2.3) $-(2.5)$ are applicable to phonons inside the frequency gap of the superlattice, i.e., $|\mu+\lambda| / 2>1 .{ }^{10}$ The energy transmission rate $T$ and reflection rate $R$ are defined by (valid for $X=Y$ )

$$
T \equiv|t|^{2}, \quad R \equiv|r|^{2}
$$

\section{B. Asymptotic forms of transmitted and reflected wave packets}

We consider the phonon wave packets scattered off the barrier realized by a periodic superlattice. The timedependent wave packet of transmitted phonons can be constructed as a superposition of the stationary solutions,

$$
\begin{aligned}
\psi_{t}(x, t) & =\int \frac{d k}{2 \pi} \phi(k) t(k) e^{i(k x-\omega t)} \\
& =\int \frac{d k}{2 \pi} \phi(k) t(k) e^{i k(x-v t)}
\end{aligned}
$$

where $v \equiv v_{X}$ is the sound velocity in the detector region (and also in the substrate) and $\phi(k)$ is the Fourier transform of the phonon displacement describing the initial packet $\psi_{i}(x, t=0)$,

$$
\phi(k)=\int d x e^{-i k x} \psi_{i}(x, 0) .
$$

The phonon packet $\phi(k)$ in the wave-vector space ( $k$ space) is assumed to have a peak at $k=k_{0}$. The in- 
tensity of the transmitted wave packet is defined by

$$
I_{t}(x, t) \equiv\left|\psi_{t}(x, t)\right|^{2} .
$$

Similarly, the reflected wave packet can be constructed as

$$
\psi_{r}(x, t)=\int \frac{d k}{2 \pi} \phi(k) r(k) e^{-i k(x+v t)}
$$

and the corresponding intensity is

$$
I_{r}(x, t) \equiv\left|\psi_{r}(x, t)\right|^{2} .
$$

The above expressions can be used only asymptotically. This means that these formulas are valid only if the packets are well separated from the barrier region.

\section{Asymptotic phase times}

Now, we derive the expression of the asymptotic phase times for phonon packets defined in Sec. IIB. The phase time (the transmission time), i.e., the time that it takes the peak of a wave packet to appear on the other side of the barrier, as given by the stationary phase method, was originally introduced by Wigner for electrons tunneling through potential barriers. ${ }^{11-13}$ The phase time related to the reflected packet (the reflection time) is also introduced in a similar way.

We write the transmitted and reflected phonon packets defined in Eqs. (2.11) and (2.14) as

$$
\begin{aligned}
& \psi_{t}(x, t)=\int \frac{d k}{2 \pi} \phi(k)|t(k)| e^{i(k x-\omega t+\alpha)}, \\
& \psi_{r}(x, t)=\int \frac{d k}{2 \pi} \phi(k)|r(k)| e^{-i(k x+\omega t-\beta)} .
\end{aligned}
$$

In these equations we have introduced the phases $\alpha(k)$ and $\beta(k)$ of the transmitted and reflected amplitudes defined by $t(k)=|t(k)| e^{i \alpha(k)}, r(k)=|r(k)| e^{i \beta(k)}$. With the use of Eqs. (2.1) and (2.2), these phases are written more explicitly as

$$
\tan \alpha=\frac{h_{-} S(N) \cos (k N D)-2 C(N) \sin (k N D)}{h_{-} S(N) \sin (k N D)+2 C(N) \cos (k N D)},
$$

and

$$
\tan \beta=-\frac{(\lambda-\mu) h_{-} S(N)+2 h_{+} C(N)}{h_{+} h_{-} S(N)-2(\lambda-\mu) C(N)}
$$

where

$$
h_{ \pm}=\left(Z_{X} / Z_{A}\right) \sigma \pm\left(Z_{A} / Z_{X}\right) \zeta
$$

In the stationary phase approximation, the dominant contributions to the integrals of (2.16) and (2.17) come from the regions around $k$ 's satisfying

$$
\frac{d \alpha}{d k}+x-\frac{d \omega}{d k} t=0, \quad \text { and } \quad-\frac{d \beta}{d k}+x+\frac{d \omega}{d k} t=0,
$$

respectively. From Eq. (2.21) we see that the peaks of the transmitted and reflected packets move according to

$$
x=v\left(t-\left.\frac{d \alpha}{d \omega}\right|_{k_{0}}\right), \quad x=-v\left(t-\left.\frac{d \beta}{d \omega}\right|_{k_{0}}\right) .
$$

Thus, the scattering by the barrier causes temporal delays for the transmitted and reflected packets,

$$
\tau^{t}=\left.\frac{d \alpha}{d \omega}\right|_{k_{0}} \quad \text { and } \quad \tau^{r}=\left.\frac{d \beta}{d \omega}\right|_{k_{0}}
$$

which can be calculated from Eqs. (2.18) and (2.19). Here, we have followed the motions of the peaks of the narrow phonon packets and derived the formal expressions of the phase times, Eq. (2.23). In the electron tunneling problem, Hauge et al. ${ }^{7,8}$ examined in detail the asymptotic time dependence of the centers of gravity of the transmitted and reflected wave packets and derived the corrections to Eq. (2.23). Those corrections are important for the packets with large widths in the $k$ space. In the present study, however, we consider the case where the phonon packets have finite but sufficiently small widths in the $k$ space for which Eq. (2.23) holds in a good approximation.

\section{Approximate expressions for the phase times}

Here, we derive approximate expressions for the phase times which are useful in understanding the physics involved. (We further assume $X=A$, for simplicity.) Suppose that the number of periods of the superlattice is large, so that $e^{N \theta} \gg e^{-N \theta}$ is satisfied, where $\theta$ is the positive solution of Eq. (2.5). In this case, $\sinh N \theta \sim \cosh N \theta \sim e^{N \theta} / 2$, and the transmission amplitude, Eq. (2.1), becomes

$$
t=( \pm 1)^{N} 2 e^{-i k L} e^{-N \theta}\left[1 \mp\left(\frac{\sigma-\zeta}{2 \sinh \theta}\right) i\right]^{-1}
$$

where the upper sign corresponds to the case $(\mu+\lambda) / 2>$ 1 and the lower sign to the case $(\mu+\lambda) / 2<-1$, and $L=N D$ is the thickness of the superlattice. It should be noted that the factor $e^{-i k L}$ in Eq. (2.24) is a kinematical one characteristic of the transmitted wave and the exponentially small factor $e^{-N \theta}$ is indicative of Bragg reflection. The phase of the transmission amplitude is calculated from Eq. (2.24) as

$$
\alpha= \pm \tan ^{-1}\left(\frac{\sigma-\zeta}{2 \sinh \theta}\right)-k L
$$

Similarly, for the reflected phonon packet, we obtain

$$
r=\frac{\sigma+\zeta-i(\lambda-\mu)}{\sigma-\zeta \pm 2 i \sinh \theta}
$$

$$
\beta=\frac{d_{A}}{v_{A}} \omega \pm \tan ^{-1}\left(\frac{\sigma-\zeta}{2 \sinh \theta}\right)-\frac{\pi}{2}
$$


The first term of Eq. (2.27) arises from the fact that we have chosen $X=A$. If we choose $X=B, d_{A} \omega / v_{A}$ is replaced by $-d_{B} \omega / v_{B}$. Thus, this term represents the effect of the boundary between the substrate and the superlattice. The second term which also exists in the expression of $\alpha$ is attributed to the periodic structure.

Now, we focus on the frequency gap due to the first order Bragg reflection where $(\mu+\lambda) / 2<-1$ holds. We define the frequency $\omega_{0}$ at the center of this frequency gap by the equation $\cos \left(k_{A} d_{A}+k_{B} d_{B}\right)=-1$. Explicitly,

$$
\omega_{0}=\pi\left(\frac{d_{A}}{v_{A}}+\frac{d_{B}}{v_{B}}\right)^{-1} .
$$

Inside the frequency gap, $\theta$ defined by Eq. (2.5) is close to zero and changes very slowly. Thus, putting $\theta \cong \theta_{0} \equiv$ $\theta\left(\omega_{0}\right)$, we have an approximated expression for $\theta_{0}$ (see Appendix A)

$$
\theta_{0}=\frac{\left|Z_{A}-Z_{B}\right|}{\sqrt{Z_{A} Z_{B}}}\left|\sin \left(\frac{d_{A}}{v_{A}} \omega_{0}\right)\right| .
$$

Next, we consider a frequency $\omega$ close to $\omega_{0}$. Keeping terms up to the first order in $\omega-\omega_{0}$, we find from Eqs. (2.7) and (2.8),

$$
\begin{aligned}
\zeta-\sigma= & -2 \sin \left[\left(\frac{d_{A}}{v_{A}}+\frac{d_{B}}{v_{B}}\right) \omega\right] \\
& -\frac{\left(Z_{A}-Z_{B}\right)^{2}}{Z_{A} Z_{B}} \cos \left(\frac{d_{A}}{v_{A}} \omega_{0}\right) \sin \left(\frac{d_{B}}{v_{B}} \omega_{0}\right) \\
\cong & \frac{2 \pi}{\omega_{0}}\left(\omega-\omega_{0}\right)
\end{aligned}
$$

where we have assunied that $\left(Z_{A}-Z_{B}\right)^{2} / Z_{A} Z_{B}$ is much smaller than unity. Since $\sinh \theta \sim \theta_{0}$, we get

$$
\tan ^{-1}\left(\frac{\zeta-\sigma}{2 \sinh \theta}\right) \cong \frac{\pi}{\theta_{0} \omega_{0}}\left(\omega-\omega_{0}\right)
$$

This equation is valid for $\omega$ close to $\omega_{0}$ satisfying $\omega-\omega_{0} \ll$ $\theta_{0} \omega_{0} / \pi$. Finally, inserting Eq. (2.31) into Eqs. (2.25) and (2.27), we have the expressions for the phases $\alpha$ and $\beta$ at $\omega \simeq \omega_{0}$

$$
\begin{aligned}
& \alpha=\frac{\pi}{\theta_{0} \omega_{0}}\left(\omega-\omega_{0}\right)-\frac{L}{v} \omega, \\
& \beta=\frac{d_{A}}{v_{A}} \omega+\frac{\pi}{\theta_{0} \omega_{0}}\left(\omega-\omega_{0}\right)-\frac{\pi}{2},
\end{aligned}
$$

and the phase times become

$$
\begin{aligned}
\tau_{1}^{t} & =\frac{d \alpha}{d \omega} \\
& =\frac{\sqrt{Z_{A} Z_{B}}}{\left|Z_{A}-Z_{B}\right|}\left|\sin \left(\frac{d_{A}}{v_{A}} \omega_{0}\right)\right|^{-1}\left(\frac{d_{A}}{v_{A}}+\frac{d_{B}}{v_{B}}\right)-\frac{L}{v},
\end{aligned}
$$

$$
\begin{aligned}
\tau_{1}^{r}=\frac{d \beta}{d \omega} \cong & \frac{d_{A}}{v_{A}}+\frac{\sqrt{Z_{A} Z_{B}}}{\left|Z_{A}-Z_{B}\right|}\left|\sin \left(\frac{d_{A}}{v_{A}} \omega_{0}\right)\right|^{-1} \\
& \times\left(\frac{d_{A}}{v_{A}}+\frac{d_{B}}{v_{B}}\right) .
\end{aligned}
$$

As we shall show in the next subsection these expressions for the delay times are in good agreement with those obtained by the numerical calculations using the exact formulas, Eqs. (2.18) and (2.19). From Eq. (2.35), it is seen that $\tau_{1}^{r}$ for the reflected packet depends only on the parameters describing the constituent layers and takes a positive value, that is, the time delay is always found for the reflected phonon packet. For the transmitted packet, however, the width of the barrier $L=N\left(d_{A}+d_{B}\right)$ plays an important role in determining $\tau_{1}^{t}$. For a large number of period $N$ Eq. (2.34) becomes negative and the time advance should be found. However, as $N$ increases the transmission rate decreases as $T \sim e^{-2 N \theta}$ [see Eq. (2.24)], so the phonon packet cannot be transmitted effectively through the system.

\section{E. Numerical example}

As a numerical example we consider a superlattice consisting of GaAs $(A)$ and AlAs $(B)$ layers. Figure 1 illustrates the frequency dependence of the phonon transmission rate in the (100)AlAs/GaAs superlattice assumed. In this example, the unit period is $(\mathrm{AlAs})_{15}(\mathrm{GaAs})_{15}$ $(D=85 \AA$ ) and the number of periods is $N=12$, or $L=1020 \AA$. The substrate $(X)$ and detector layer $(Y)$ are assumed to be $\mathrm{GaAs}$, for simplicity. A large transmission dip in Fig. 1 corresponds to the lowest frequency gap (phonon stop band) associated with the first-order Bragg reflection of phonons in the present system. The frequencies inside this frequency gap are determined from the condition $(\mu+\lambda) / 2<-1$ and their range is 286 to

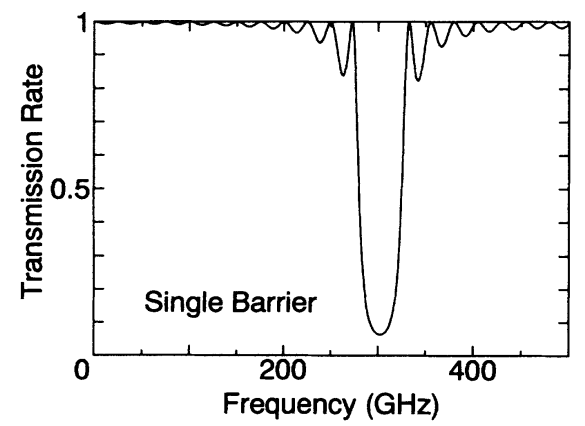

FIG. 1. Frequency dependence of the phonon transmission rate $T=|t|^{2}$ in a (100)AlAs/GaAs superlattice. The assumed unit period is $(\mathrm{AlAs})_{15}(\mathrm{GaAs})_{15}$ and the number of periods is $N=12$. The parameters used are as follows: The thickness of one monolayer is $2.83 \AA$ in the (100) direction for both GaAs and AlAs; the mass densities and longitudinal sound velocities are $5.36 \mathrm{~g} / \mathrm{cm}^{3}$ and $4.71 \mathrm{~km} / \mathrm{s}$ for $\mathrm{GaAs}$, and $3.76 \mathrm{~g} / \mathrm{cm}^{3}$ and $5.65 \mathrm{~km} / \mathrm{s}$ for AlAs. This figure covers the frequency range where only the transmission dip due to the first-order Bragg reflection appears. 
$318 \mathrm{GHz}$. The transmission and reflection rates within this frequency gap are enlarged and shown in Fig. 2.

We assume that the initial wave packet defined in the substrate has the Gaussian form

$$
\psi_{i}(x, 0)=\exp \left[-\frac{\left(x-x_{0}\right)^{2}}{4(\Delta x)^{2}}+i k_{0} x\right]
$$

where $x_{0}$ is the coordinate at the center of the initial packet. With this form of the packet, the Fourier component $\phi(k)$ is given by

$$
\phi(k)=\frac{2 \sqrt{\pi}}{\Delta k} \exp \left[-\frac{\left(k-k_{0}\right)^{2}}{(\Delta k)^{2}}+i\left(k_{0}-k\right) x_{0}\right],
$$

where $\Delta k=1 /(\Delta x)$. We choose $\nu_{0}=\omega_{0} / 2 \pi=v k_{0} / 2 \pi=$ $302.0 \mathrm{GHz}$ (the frequency at the minimum of the transmission rate), and $\Delta \nu=v \Delta k / 2 \pi=10.0 \mathrm{GHz}$ (sound velocity $v$ of the longitudinal mode is $4.71 \mathrm{~km} / \mathrm{s}$ in GaAs). Hereafter, we put $x_{0}=0$. The corresponding $|\phi(k)|^{2}$ is shown in Fig. 2, i.e., $\phi(k)$ is finite within the frequency window corresponding to the gap we consider.

Numerically integrating Eqs. (2.11) and (2.14), we have obtained the intensities of the transmitted and reflected wave packets as shown in Fig. 3. If the superlattice is absent, the reflected packet does not exist and the center of the incident, or transmitted packet is located at $x=v t$, which corresponds to the origin of this figure. The existence of the barrier introduces both the transmitted and reflected packets (moving to the right and left, respectively) as well as their time delay or advance. From this figure, we can measure the spatial delay (advance) of the packets $(\sim 500 \AA)$ equivalent to the corresponding time delay (advance). Thus, we find the time advance for the transmitted wave packet and the time delay for the reflected packet and their magnitudes are about 10 ps. To examine these features in more detail, we have plotted in Fig. 4(a) the phases $\alpha$ and $\beta$ of the transmission and reflection amplitudes calculated numerically from Eqs. (2.18) and (2.19) (solid lines) and also

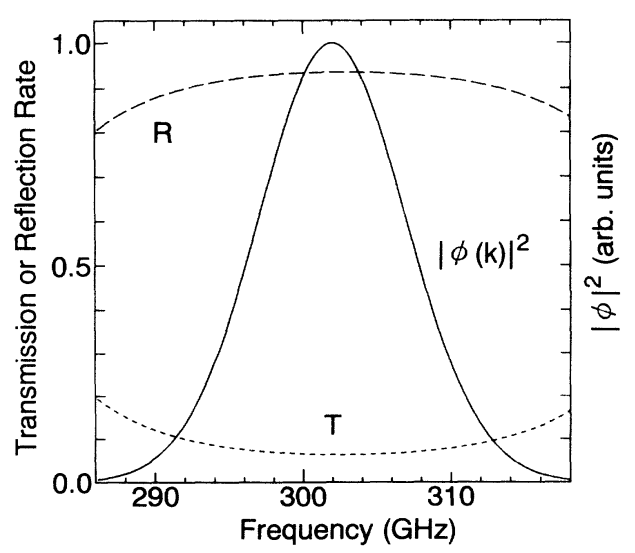

FIG. 2. Phonon transmission rate $T=|t|^{2}$ (dashed line) and reflection rate $R=|r|^{2}$ (broken line) within the lowest-frequency gap $(286-318 \mathrm{GHz})$ of the single-barrier system (same as Fig. 1). $\phi$ is the Fourier component of the assumed initial wave packet $\psi_{i}$. Note that $\phi$ is finite within this frequency gap.

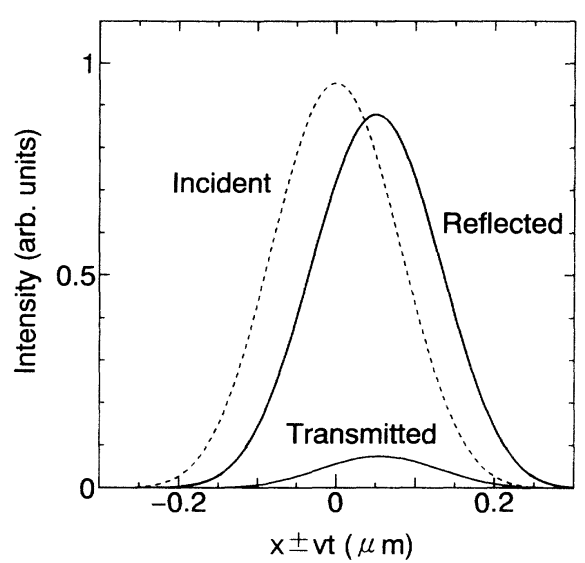

FIG. 3. Asymptotic forms of the transmitted (thin line) and reflected (bold line) intensities of the phonon wave packets in the single-barrier system which are compared to the intensity of the initial packet (dashed line). The distance between the peaks of the incident and transmitted (reflected) packets shows the spatial advance (delay) of the transmitted (reflected) packet. The incident and transmitted packets move toward $+x$ direction and the reflected packet moves toward $-x$ direction. Thus, $x$ and $t$ satisfying $x-v t=\operatorname{const}(x+v t=$ const $)$ give an equal phase for the transmitted (reflected) packet.

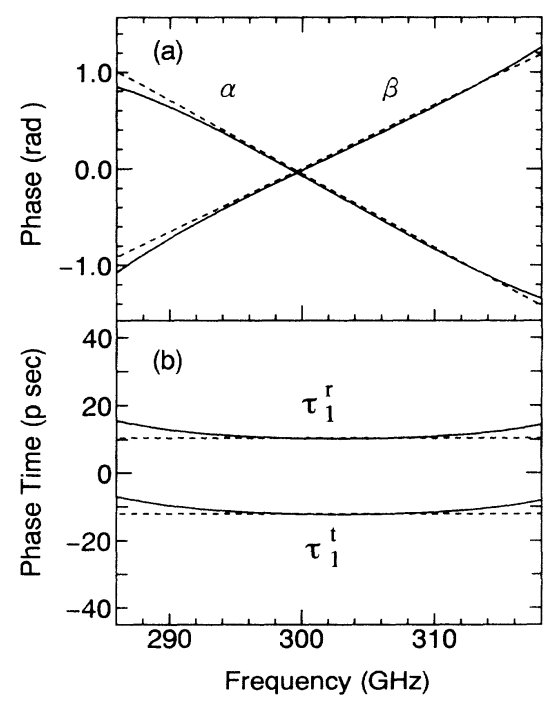

FIG. 4. (a) Phases versus frequency of the transmitted and reflected amplitudes of phonons scattered off the single-barrier system. The solid lines are calculated from the exact expressions [Eqs. (2.18) and (2.19)] and the dashed lines are calculated based on the approximate expressions [Eqs. (2.32) and (2.33)]. (b) Phase times of the transmitted and reflected phonons in the single-barrier system. The solid lines are calculated from the exact formulas [Eqs. (2.23) with Eqs. (2.18) and (2.19)]. The dashed lines are calculated based on the approximate expressions [Eqs. (2.34) and (2.35)]. 
from the approximated formula Eqs. (2.32) and (2.33) (dashed lines). The corresponding phase times $\tau^{t}$ and $\tau^{r}$ calculated are shown in Fig. 4(b). As expected the time delay (advance) for the reflected (transmitted) wave is about 10 ps for phonons within the frequency gap considered.

The origin of these time advance and delay is the interference between the forward and backward propagating phonons in the barrier region. The interference generally induces the attenuation of waves due to phase incoherence. However, the wave front of the incident packet is not suffered from the interference though its later part is suffered a lot, leading to larger attenuation of the later part of the transmitted packet and the output peak shifted towards earlier time as the barrier thickness increases. This causes the apparent time advance of the transmitted packet. The time delay of the reflected packet is also expected by the similar qualitative argument. As shown in Fig. 2 the frequency dependence of the transmission and reflection rates is very weak over the lowest phonon stop band, so the transmitted and reflected phonon packets $\psi_{t}$ and $\psi_{r}$ are also essentially the Gaussian for the initial packet chosen in the present example. The numerical calculations of the transmitted and reflected intensities shown in Fig. 3 support this result.

\section{DOUBLE-BARRIER SYSTEM FOR PHONONS}

In this section, we consider the transmission and reflection times of phonons through a symmetric doublebarrier system. This system is realized by a bulk material sandwiched between periodic superlattices, e.g., $X(A B)^{N} C(A B)^{N} Y$, where $X, Y$, and $C$ denote the substrate, detector, and a layer different from $A$ and $B$, re- spectively, and $N$ is the number of the periodicities of the superlattices acting as the symmetric double barriers for phonons. A characteristic feature of phonons in this structure is the presence of the resonance. The transmission characteristics of monochromatic phonons in such a system have been studied in Ref. 4 for the case that $C$ layer consists of the same material as $A$ or $B$ layer but with different thickness. The phonons in a frequency gap of the superlattices are resonantly transmitted through the entire system when the frequency of incident phonons coincides with one of the discrete frequency levels induced by the sandwiched layer. This corresponds to the resonant tunneling of electrons in a double-barrier quantumwell structure. ${ }^{5}$ In this section, we focus on the effect of the resonance on a phonon packet traveling through the double-barrier structure. First, we briefly summarize the expressions for the transmission and reflection amplitudes given in Ref. 4. Then, the phonon wave packets scattered off the double-barrier structure are examined and the expressions for the asymptotic phase times as well as the shapes of the transmitted and reflected packets are derived.

\section{A. Transmission and reflection amplitudes}

The transmission and reflection amplitudes of phonons in the double-barrier structure for $X=Y$ are given by ${ }^{14}$

$$
\begin{aligned}
& t=\frac{2 i}{\left(\frac{Z_{X}}{Z_{A}} b-\frac{Z_{A}}{Z_{X}} c\right)+i(d+a)} e^{-i k_{X} L}, \\
& r=\frac{\left(\frac{Z_{X}}{Z_{A}} b+\frac{Z_{A}}{Z_{X}} c\right)+i(d-a)}{\left(\frac{Z_{X}}{Z_{A}} b-\frac{Z_{A}}{Z_{X}} c\right)+i(d+a)}
\end{aligned}
$$

where

$$
\begin{aligned}
a & =\frac{1}{2}\left(1 \pm \frac{\lambda-\mu}{2 \sinh \theta}\right) G_{ \pm} e^{2 N \theta}+\frac{1}{2}\left(1 \mp \frac{\lambda-\mu}{2 \sinh \theta}\right) G_{\mp} e^{-2 N \theta}-\frac{(\lambda-\mu)(\zeta-\sigma)}{4 \sinh ^{2} \theta} \sin \gamma \\
b & = \pm \frac{\sigma}{2 \sinh \theta}\left(G_{ \pm} e^{2 N \theta}-G_{\mp} e^{-2 N \theta}\right)+\left(1-\frac{\sigma(\zeta-\sigma)}{2 \sinh ^{2} \theta}\right) \sin \gamma \\
c & = \pm \frac{\zeta}{2 \sinh \theta}\left(G_{ \pm} e^{2 N \theta}-G_{\mp} e^{-2 N \theta}\right)-\left(1+\frac{\zeta(\zeta-\sigma)}{2 \sinh ^{2} \theta}\right) \sin \gamma, \\
d & =\frac{1}{2}\left(1 \mp \frac{\lambda-\mu}{2 \sinh \theta}\right) G_{ \pm} e^{2 N \theta}+\frac{1}{2}\left(1 \pm \frac{\lambda-\mu}{2 \sinh \theta}\right) G_{\mp} e^{-2 N \theta}+\frac{(\lambda-\mu)(\zeta-\sigma)}{4 \sinh ^{2} \theta} \sin \gamma \\
G_{ \pm} & =\cos \gamma \pm \frac{\zeta-\sigma}{2 \sinh \theta} \sin \gamma,
\end{aligned}
$$

with $L=2 N D+D^{\prime}$ and $\gamma=k_{C} D^{\prime}$ ( $D^{\prime}$ is the thickness of $C$ layer). In Eqs. (3.3) to (3.7), the upper sign corresponds to the case $(\mu+\lambda) / 2>1$ and the lower sign to $(\mu+\lambda) / 2<-1$. The above equations are valid if the frequency of phonons is within the spectral gap of the superlattices. The phases $\alpha$ and $\beta$ of the transmission and reflection amplitudes are given by

$$
\begin{gathered}
\tan \alpha=\frac{Q_{-} \cos k_{X} L-P_{+} \sin k_{X} L}{P_{+} \cos k_{X} L+Q_{-} \sin k_{X} L} \\
\tan \beta=\frac{P_{-} Q_{-}-Q_{+} P_{+}}{Q_{+} Q_{-}+P_{+} P_{-}}
\end{gathered}
$$

where $P_{ \pm}=d \pm a$ and $Q_{ \pm}=\left(Z_{X} / Z_{A}\right) b \pm\left(Z_{A} / Z_{X}\right) c$. 
Combining Eqs. (3.1), (3.2), and (2.10), we can calculate the transmission and reflection rates of phonons inside the frequency gap of the double-barrier system.

\section{B. Approximate expressions for the phase times}

As in the single-barrier system we assume $X=A$ and suppose the case that the number of periods $N$ of the superlattices is large and $e^{2 N \theta} \gg e^{-2 N \theta}$ is satisfied. In this case, we can also derive approximate expressions for both the phases and amplitudes of the transmitted and reflected packets around the resonant frequencies. Neglecting the terms of the order of $e^{-2 N^{*} \theta}$ in Eqs. (3.3)(3.6), we have

$a+d=G_{ \pm} e^{2 N \theta}$

$b-c= \pm \frac{\sigma-\zeta}{2 \sinh \theta} G_{ \pm} e^{2 N \theta}+\left[2+\frac{(\sigma-\zeta)^{2}}{2 \sinh ^{2} \theta}\right] \sin \gamma$

By using Eqs. (3.10) and (3.11) and putting $k_{X}=k$, the transmission amplitude becomes

$$
t=\frac{1}{f(\omega)} \frac{2 e^{-i k L}}{G_{ \pm} e^{2 N \theta}-2 i \bar{f}(\omega) \sin \gamma},
$$

where

$$
f(\omega)=1 \mp i \frac{\sigma-\zeta}{2 \sinh \theta}
$$

and $\bar{f}$ is the complex conjugate of $f$. Now, we expand $G_{ \pm}(\omega) e^{2 N \theta}$ around a frequency $\omega_{1}$ satisfying $G_{ \pm}\left(\omega_{1}\right)=0$ and keep only the term linear in $\omega-\omega_{1}$,

$$
G_{ \pm}(\omega) e^{2 N \theta} \sim G_{ \pm}^{\prime}\left(\omega_{1}\right) e^{2 N \theta_{1}}\left(\omega-\omega_{1}\right),
$$

where $G_{ \pm}^{\prime}\left(\omega_{1}\right)=\left.\left(d G_{ \pm} / d \omega\right)\right|_{\omega=\omega_{1}}, \theta_{1}=\theta\left(\omega_{1}\right)$ and $\omega_{1}$ is a resonant frequency in the limit of large $N$. Keeping the terms of the order of $e^{2 N \theta_{1}}$ and 1, we find from Eq. (3.12)

$$
t=\frac{-i \Gamma}{\omega-\omega_{\text {reso }}+i \Gamma} e^{-i\left(\gamma_{1}+k L\right)},
$$

where $k_{1}=\omega_{1} / v, \gamma_{1}=\gamma\left(\omega_{1}\right)$, and we have introduced the width $\Gamma$ of the resonant peak,

$$
\frac{1}{\Gamma} \equiv \frac{-1}{2 \sin \gamma_{1}} e^{2 N \theta_{1}} G_{ \pm}^{\prime}\left(\omega_{1}\right) \text {. }
$$

and the resonant frequency

$$
\omega_{\text {reso }} \equiv \omega_{1}+\Gamma \cot \gamma_{1} \text {. }
$$

In deriving Eq.(3.15), we have used the relation [see (3.7)]

$$
\cos \gamma_{1}= \pm \frac{\sigma_{1}-\zeta_{1}}{2 \sinh \theta_{1}} \sin \gamma_{1}
$$

where $\sigma_{1}=\sigma\left(\omega_{1}\right)$ and $\zeta_{1}=\zeta\left(\omega_{1}\right)$. Here, we note that the width $\Gamma$ defined by Eq. (3.16) is positive as shown in Appendix B.
Similarly, from Eq. (3.2) we also find for the reflection amplitude

$$
r=A_{0} e^{-i \gamma_{1}}\left(1-\frac{i \Gamma}{\omega-\omega_{\text {reso }}+i \Gamma}\right)
$$

where $A_{0}=\left[i\left(\lambda_{1}-\mu_{1}\right)-\left(\zeta_{1}+\sigma_{1}\right)\right] \sin \gamma_{1} /\left(2 \sinh \theta_{1}\right)$, and $\lambda_{1}=\lambda\left(\omega_{1}\right)$ and $\mu_{1}=\mu\left(\omega_{1}\right)$. As the number of periodicities $N$ of the superlattice increases, the width of the resonant peak in transmission becomes narrow according to Eq. (3.16) and the frequency $\omega_{\text {reso }}$ approaches $\omega_{1}$. Thus, changing the number of periods, we can control the width of the resonant peak of the system.

From Eq. (3.15), the phase of the transmission coefficient becomes

$$
\alpha=-\gamma_{1}-k L-\tan ^{-1}\left(\frac{\Gamma}{\omega-\omega_{\text {reso }}}\right)+\frac{\pi}{2} .
$$

This result is valid in the vicinity of the resonance but does not reproduce the phase $\alpha$ in the form of Eq. (2.25), which is expected at a frequency outside the narrow resonance. More elaborated calculation gives

$$
\alpha= \pm \tan ^{-1}\left(\frac{\sigma-\zeta}{2 \sinh \theta}\right)-k L-\tan ^{-1}\left(\frac{\Gamma}{\omega-\omega_{\text {reso }}}\right)
$$

Here, we apply Eq. (3.21) to phonons within the lowest frequency gap and retain the lower sign in Eq. (3.21). In this case, from Eqs. (2.29) and (2.31), the phase $\alpha$ is approximated as

$$
\begin{aligned}
\alpha= & \frac{\sqrt{Z_{A} Z_{B}}}{\left|Z_{A}-Z_{B}\right|}\left|\sin \left(\frac{d_{A}}{v_{A}} \omega_{0}\right)\right|^{-1}\left(\frac{d_{A}}{v_{A}}+\frac{d_{B}}{v_{B}}\right)\left(\omega-\omega_{0}\right) \\
& -\frac{L}{v_{A}} \omega-\tan ^{-1}\left(\frac{\Gamma}{\omega-\omega_{\text {reso }}}\right)
\end{aligned}
$$

and the expression of the phase time becomes

$$
\tau_{2}^{t}=\frac{d \alpha}{d \omega}=\tau_{1}^{t}+\frac{\Gamma}{\left(\omega-\omega_{\text {reso }}\right)^{2}+\Gamma^{2}}
$$

The first term of Eq. (3.23) is identical to the phase time in the single-barrier system [Eq. (2.34)] and the second term has the form characteristic of the resonance.

Similarly, for the reflected phonons, we obtain

$$
\beta=\frac{d_{A}}{v_{A}} \omega \pm \tan ^{-1}\left(\frac{\sigma-\zeta}{2 \sinh \theta}\right)-\tan ^{-1}\left(\frac{\Gamma}{\omega-\omega_{\text {reso }}}\right) .
$$

In the lowest-frequency gap, this phase is reduced to

$$
\begin{aligned}
\beta= & \frac{d_{A}}{v_{A}} \omega+\frac{\sqrt{Z_{A} Z_{B}}}{\left|Z_{A}-Z_{B}\right|}\left|\sin \left(\frac{d_{A}}{v_{A}} \omega_{0}\right)\right|^{-1} \\
& \times\left(\frac{d_{A}}{v_{A}}+\frac{d_{B}}{v_{B}}\right)\left(\omega-\omega_{0}\right)-\tan ^{-1}\left(\frac{\Gamma}{\omega-\omega_{\text {reso }}}\right)+\frac{\pi}{2} .
\end{aligned}
$$


From this equation, we have the similar expression for the phase time for the reflected phonons:

$$
\tau_{2}^{r}=\frac{d \beta}{d \omega}=\tau_{1}^{r}+\frac{\Gamma}{\left(\omega-\omega_{\text {reso }}\right)^{2}+\Gamma^{2}} .
$$

As in the case of the transmission the extra time delay associated with the resonance is added to $\tau_{1}^{r}$ in the single barrier system. Equations (3.23) and (3.26) tell us that the maximum of the time delay $\sim 1 / \Gamma$ is obtained at the resonant frequency $\omega=\omega_{\text {reso }}$ for both the transmitted and reflected packets and its magnitude increases as the width of the resonance becomes smaller, or equivalently the number of periods of the superlattices becomes larger. This indicates that the time delay in the double-barrier structure is yielded as the result of the finite lifetime of the resonant level (or the time for phonons trapped in the layer between the barriers) in addition to the delay induced by the barrier itself.

\section{Numerical example}

For a numerical example, we assume that two periodic superlattices, i.e., symmetric double barriers for phonons, have the same structures as the one used for the singlebarrier case considered in the previous section. The sandwiched layer $C$ is assumed to be AlAs and its thickness $D^{\prime}$ is the same as the size of each superlattice, i.e., $D^{\prime}=N D$ and $L=3 N D$. Figure 5 shows the frequency dependence of the phonon transmission rate through the assumed structure. We find two sharp peaks (at about $293 \mathrm{GHz}$ and $309 \mathrm{GHz}$ ) within the lowest-frequency gap of the superlattice. These peaks in transmission stem from the resonances characteristic of the double-superlattice system. The transmission rate around the lowest resonant peak is enlarged in Fig. 6.

We examine the time evolution of the phonon wave packet whose average wave number in the initial state coincides with the resonant frequency. As in the singlebarrier system, we assume the Gaussian form [Eq. (2.37)] for the incident wave packet. Numerically, $\nu_{0}=\nu_{\text {reso }}=$

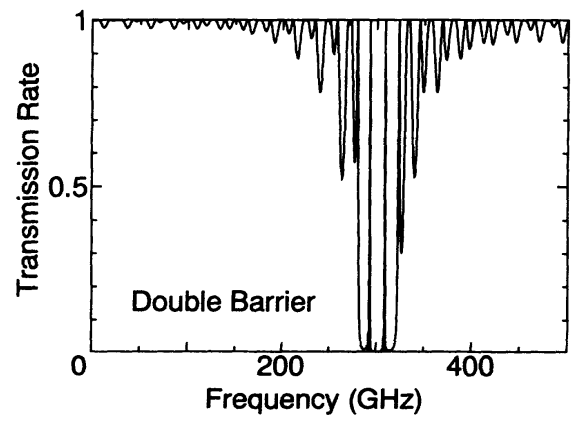

FIG. 5. Frequency dependence of phonon transmission rate in the double-barrier system. The superlattices assumed are the same as in Fig. 1 and an AlAs layer with the same thickness as that of the single superlattice is sandwiched between the superlattice barriers.

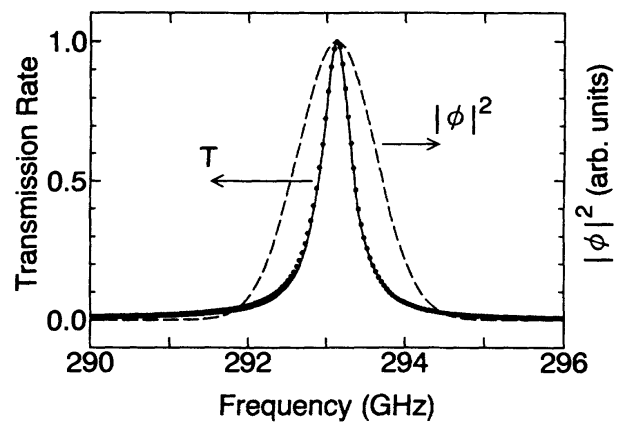

FIG. 6. Phonon transmission rate $\mathrm{T}=|t|^{2}$ (solid line) versus frequency around the lowest resonant frequency (293.2 $\mathrm{GHz}$ ) in the double-barrier system. The dots are the transmission rate calculated based on the approximate expressions (3.15). The broken line shows the square of the Fourier component $\phi$ of the initial wave packet, where the frequency at the peak of $\phi$ is chosen to $\omega_{\text {reso. }}$.

$293.2 \mathrm{GHz}$, and $\Delta \nu=5.0 \mathrm{GHz}$. Figure 6 also shows the corresponding frequency distribution $\phi(k)$. In this example, the width of the chosen packet in the frequency domain is larger than the intrinsic width of the doublebarrier resonance but both the transmitted and reflected packets are sizable.

The intensities of the transmitted and reflected packets calculated with the exact expressions for the transmission and reflection amplitudes (3.1) and (3.2) are shown in Fig. 7. It is seen that the asymptotic forms of the transmitted and reflected phonon packets are quite different from that of the incident packet. One of the most noticeable features is that the transmitted packet has a long tail followed by the peak, which decreases exponentially with a time constant $\sim 0.69 \mathrm{~ns}$.

The reflected packet exhibits the similar behavior as

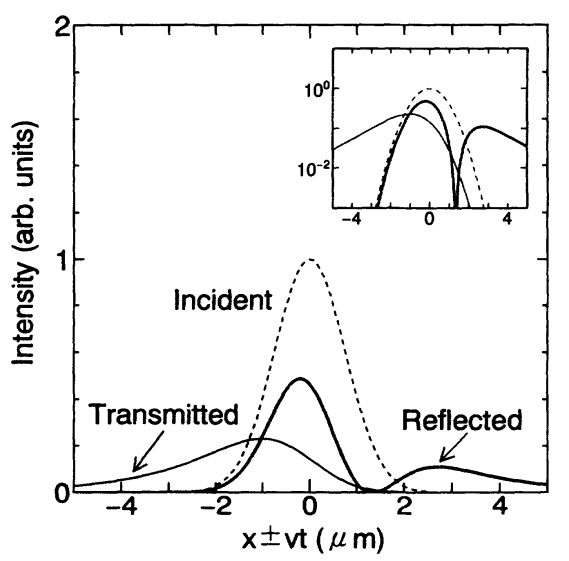

FIG. 7. Asymptotic forms of the transmitted (thin line) and reflected (bold line) intensities of the phonon wave packets in the double-barrier structure calculated from the exact expressions [Eqs. (2.16) and (2.17) with (3.1) and (3.2)] which are compared to that of the Gaussian initial packet (dashed line). Inset shows the logarithmic plot of these intensities. 


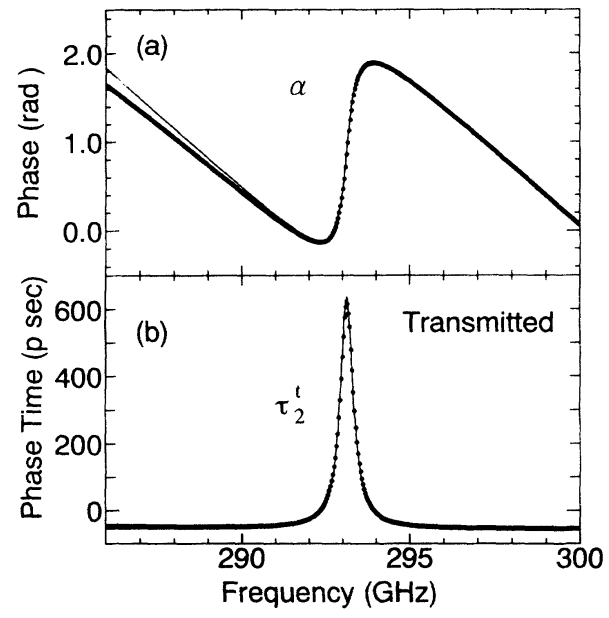

FIG. 8. Phases (a) and phase time (b) versus frequency of the transmitted phonons scattered off the double-barrier system. The solid lines are calculated from the exact expressions [Eqs. (2.23) and (3.8)] and the dots are calculated based on the approximate expressions [Eqs. (3.22) and (3.23)].

the transmitted packet but it has double peaks. The same kind of features are also found in the simulations of the resonant tunneling of electrons. ${ }^{15}$ The long tails associated with the resonant phonons are interpreted as the result of multiple reflections from double barriers of phonons trapped within the sandwiched layer. The appearance of the double peaks for the reflected packet is explained based on the analytical calculation given in the next subsection.

Figures 8 and 9 show the phases $\alpha$ and $\beta$ and the corresponding phase times $\tau_{2}^{t}$ and $\tau_{2}^{r}$. We find large time delays of the packets around the resonant frequency, which become about $0.6 \sim 0.7 \mathrm{~ns}$ in the present example, much

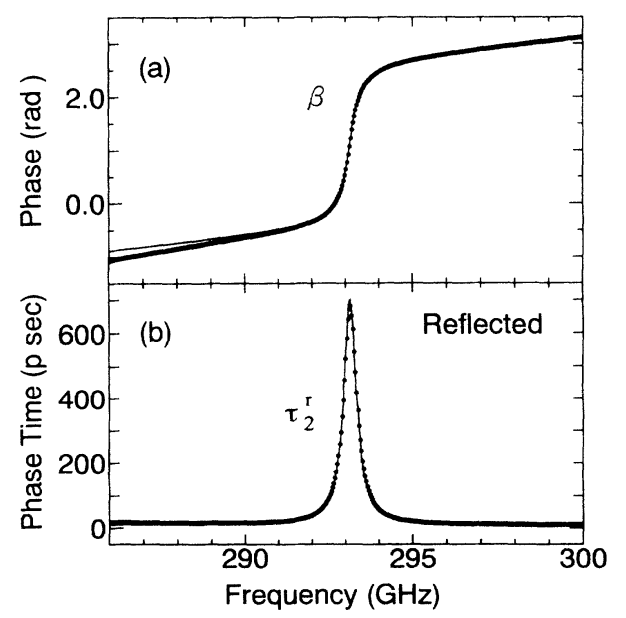

FIG. 9. Phase (a) and phase time (b) versus frequency of the reflected phonons in the double-barrier system. The solid lines are calculated from the exact expressions [Eqs. (3.26) and (3.9)] and dots are calculated based on the approximate expressions [Eqs. (3.25) and (3.26)]. larger than the phase times $\tau_{1}^{t}$ and $\tau_{1}^{r}$ for the singlebarrier system.

\section{Expressions for the amplitudes of the transmitted and reflected packets}

As we have seen, the asymptotic shapes of the transmitted and reflected packets scattered off the double barriers are quite different from the shape of the incident packet. Here we consider the shapes of these packets by analytically calculating $\psi_{t}$ and $\psi_{r}$ with the transmission and reflection amplitudes (3.15) and (3.19). Assuming the Gaussian form Eq. (2.37) with $k_{0}=k_{\text {reso }} \equiv \omega_{\text {reso }} / v$ for the initial packet, we can express the transmitted and reflected packets as

$$
\psi_{t}=\frac{-i \tilde{\Gamma}}{\sqrt{\pi} \Delta k} e^{-i \gamma_{1}} \int_{-\infty}^{\infty} \frac{e^{-\left(k-k_{\mathrm{reso}}\right)^{2} /(\Delta k)^{2}}}{k-k_{\mathrm{reso}}+i \tilde{\Gamma}} e^{i k(x-v t-L)} d k,
$$

$$
\begin{aligned}
\psi_{r}= & \frac{A_{0}}{\sqrt{\pi} \Delta k} e^{-i \gamma_{1}} \int_{-\infty}^{\infty}\left(1-\frac{i \tilde{\Gamma}}{k-k_{\text {reso }}+i \tilde{\Gamma}}\right) \\
& \times e^{-\left(k-k_{\text {reso }}\right)^{2} /(\Delta k)^{2}} e^{-i k(x+v t)} d k
\end{aligned}
$$

where $\tilde{\Gamma}=\Gamma / v$. The integrals in these equations are performed analytically and the results are expressed in terms of the complementary error function as shown in Appendix C,

$$
\begin{aligned}
\psi_{t}= & -\frac{\sqrt{\pi} \tilde{\Gamma}}{\Delta k} e^{i\left(k_{\mathrm{reso}} x_{t}-\gamma_{1}\right)} e^{\tilde{\Gamma}^{2} /(\Delta k)^{2}+\tilde{\Gamma} x_{t}} \\
& \times \operatorname{erfc}\left(\tilde{\Gamma} / \Delta k+\Delta k x_{t} / 2\right)
\end{aligned}
$$

$$
\begin{aligned}
\psi_{r}= & A_{0} e^{-i\left(k_{\text {reso }} x_{r}+\gamma_{1}\right)}\left[e^{-\left(\Delta k x_{r}\right)^{2} / 4}\right. \\
& \left.-\frac{\sqrt{\pi} \tilde{\Gamma}}{\Delta k} e^{\tilde{\Gamma}^{2} /(\Delta k)^{2}-\tilde{\Gamma} x_{r}} \operatorname{erfc}\left(\tilde{\Gamma} / \Delta k-\Delta k x_{r} / 2\right)\right]
\end{aligned}
$$

where $x_{t}=x-v t-L$ and $x_{r}=x+v t$. The intensities $I_{t}=\left|\psi_{t}\right|^{2}$ and $I_{r}=\left|\psi_{r}\right|^{2}$ calculated from Eqs. (3.29) and (3.30) are plotted in Fig. 10. They coincide well with the numerical results with the exact expressions for the transmission and reflection amplitudes. Noting that $\operatorname{erfc}(x) \sim e^{-x^{2}} /(\sqrt{\pi} x)$ for a large $x[(\mathrm{C} 9)]$, we see that for large negative $x_{t}$ and positive $x_{r}$ both $\psi_{t}$ and $\psi_{r}$ decay exponentially with the same slope given by $\tilde{\Gamma}$ (see the inset of Fig. 7). This can be physically understood as follows: the frequency distribution of the incident packet covering $\omega_{\text {reso }}$ induces a resonant state of phonons within the layer sandwiched between double barriers. This state is not stable due to the small but finite width and decays with the time constant $\Gamma^{-1}$ by emitting the phonons of the same frequency $\omega_{\text {reso }}$ in the forward and backward 


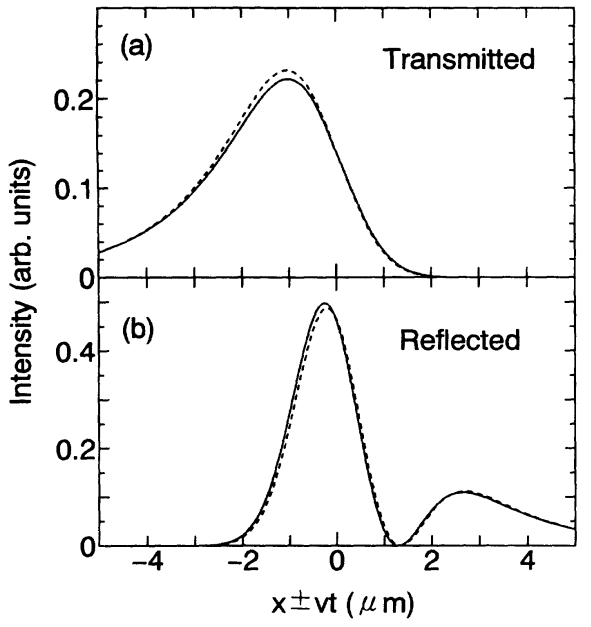

FIG. 10. Comparison of the approximated intensities of (a) transmitted and (b) reflected packets in the double-barrier system (dashed lines) with the exact results calculated from Eqs. (3.1) and (3.2) (solid lines).

directions. Thus the time delay of $\sim 1 / \Gamma$ at the resonant frequency is reflected to the slope of the intensities of both the transmitted and reflected packets rather than the positions of their peaks.

The double-peak structure in $I_{r}=\left|\psi_{r}\right|^{2}$ arises from the fact that the reflection rate has a sharp dip at the resonant frequency. More explicitly, the shape of the reflected packet in the coordinate space should be the initial Gaussian minus the transmitted packet decreasing exponentially with the decay length $1 / \tilde{\Gamma}$. The spatial decrease of the latter function is much slower than the Gaussian and hence the reflected packet has a zero, giving rise to the double-peak structure for the reflected phonon intensity.

\section{SUMMARY AND CONCLUDING REMARKS}

In the present work, we have theoretically studied the asymptotic phase times of the phonon packets propagating through the one-dimensional systems with elastic barriers consisting of semiconductor superlattices. Both the single- and double-barrier structures are considered. The analytical expressions for the transmission and reflection times for the phonon packets in these systems are derived. We have found the time advance for the transmitted wave packet (when the barrier thickness is appropriately large) and the time delay for the reflected packet in the single-barrier system. In the double-barrier system, we find large time delays for both the transmitted and reflected packets when the frequency distribution of the initial packet in the $k$ space involves the resonant frequency. The numerical values of these time delays in the double-barrier structure are found to be $0.1 \sim 1 \mathrm{~ns}$ for the system of $0.1-\mu \mathrm{m}$ dimension. These magnitudes suggest the possibility for observing of the time delays in the double-barrier structure by a phonon generation and detection experiment utilizing picosecond laser technique. ${ }^{16}$
Finally, we remark that the measurement of tunneling times of photons through a one-dimensional photonic band material similar to the single-barrier system for phonons considered here has been reported recently. The measured time advance $\sim 1.5$ fs for 1.1- $\mu$ m-thick barrier has been explained in terms of the phase time of the transmitted light pulse. ${ }^{17}$

\section{ACKNOWLEDGMENTS}

This work was supported in part by the Ogasawara Foundation for the Promotion of Science \& Engineering and a Grant-in-Aid for Scientific Research from the Ministry of Education, Science, and Culture, Japan (Grant No. 05650002).

\section{APPENDIX A}

Here we derive Eq. (2.29). For $\omega=\omega_{0}$ [the frequency at the center of the lowest frequency gap given by Eq.(2.28)], Eq. (2.5) together with Eqs. (2.6) and (2.9) becomes

$$
\begin{aligned}
-\cosh \theta_{0}= & -1-\frac{1}{2} \theta_{0}^{2} \\
= & \left.\cos \left(k_{A} d_{A}+k_{B} d_{B}\right)\right|_{\omega=\omega_{0}} \\
& -\left.\frac{\left(Z_{A}-Z_{B}\right)^{2}}{2 Z_{A} Z_{B}} \sin \left(k_{A} d_{A}\right) \sin \left(k_{B} d_{B}\right)\right|_{\omega=\omega_{0}} \\
= & -1-\left.\frac{\left(Z_{A}-Z_{B}\right)^{2}}{2 Z_{A} Z_{B}} \sin ^{2}\left(k_{A} d_{A}\right)\right|_{\omega=\omega_{0}} . \quad(\mathrm{A} 1)
\end{aligned}
$$

Hence,

$$
\begin{aligned}
\theta_{0} & =\left|\frac{Z_{A}-Z_{B}}{\sqrt{Z_{A} Z_{B}}} \sin \left(k_{A} d_{A}\right)\right|_{\omega=\omega_{0}} \mid \\
& =\left|\frac{Z_{A}-Z_{B}}{\sqrt{Z_{A} Z_{B}}} \sin \left(\frac{d_{A}}{v_{A}} \omega_{0}\right)\right| .
\end{aligned}
$$

\section{APPENDIX B}

We show $\Gamma$ (width of the resonance) defined by Eq. (3.16) is positive in the lowest-frequency gap. In this frequency range, $G_{-}$is relevant in Eq. (3.16) and $G_{-}^{\prime}\left(\omega_{1}\right)$ is calculated as

$$
G_{-}^{\prime}\left(\omega_{1}\right)=-\frac{D^{\prime}}{v_{C} \sin \gamma_{1}}-\left(\frac{\zeta-\sigma}{2 \sinh \theta}\right)_{\omega=\omega_{1}}^{\prime} \sin \gamma_{1},
$$

where we have used the relation $G_{-}\left(\omega_{1}\right)=0$. At a frequency $\omega$ around the frequency $\omega_{0}$ defined by Eq. (2.28), Eq. (2.30) and $\sinh \theta \sim \theta_{0}$ hold and we have

$$
\frac{\zeta-\sigma}{2 \sinh \theta} \cong \frac{\pi}{\theta_{0} \omega_{0}}\left(\omega-\omega_{0}\right) \text {. }
$$


Thus, we find the expression for the width of the resonant peak,

$$
\frac{1}{\Gamma}=\frac{1}{2} e^{2 N \theta_{1}}\left(\frac{D^{\prime}}{v_{C} \sin ^{2} \gamma_{1}}+\frac{\pi}{\theta_{0} \omega_{0}}\right)>0 .
$$

\section{APPENDIX C}

In this appendix we shall calculate the following integral $I$ which is used for deriving Eqs. (3.29) and (3.30):

$$
\begin{aligned}
I & =\int_{-\infty}^{\infty} \frac{1}{k-k_{0}+i b} e^{-a^{2}\left(k-k_{0}\right)^{2}} e^{i k x} d k \\
& =\exp \left[i k_{0} x-\frac{x^{2}}{4 a^{2}}\right] \int_{-\infty}^{\infty} \frac{1}{k+i b} e^{-a^{2}(k-i K)^{2}} d k \\
& \equiv \exp \left[i k_{0} x-\frac{x^{2}}{4 a^{2}}\right] J
\end{aligned}
$$

where $a, b>0, K=x /\left(2 a^{2}\right)$ and

$$
J=\int_{-\infty}^{\infty} \frac{1}{k+i b} e^{-a^{2}(k-i K)^{2}} d k .
$$

The integral $J$ is calculated by considering the contour integral in the complex $k$ plane

$$
L=\int_{C} \frac{e^{-a^{2} k^{2}}}{k+i(b+K)} d k,
$$

where the contour $C$ is the rectangle with four corners at $(R, 0),(-R, 0),(-R,-i K),(R,-i K)$ and the integral should be taken in counterclockwise if $K>0$ but clockwise if $K<0$. The integrals along two vertical paths connecting $(-R, 0)$ to $(-R,-i K)$ and $(R,-i K)$ to $(R, 0)$ vanish in the limit of $R \rightarrow \infty$. Thus, for $R \rightarrow \infty$

$$
\begin{aligned}
L & =J-\int_{-\infty}^{\infty} \frac{e^{-a^{2} k^{2}}}{k+i(b+K)} d k \\
& =\left\{\begin{array}{lll}
-2 \pi i e^{a^{2}(b+K)^{2}} & \text { for } & K<-b \\
0 & \text { for } & K>-b .
\end{array}\right.
\end{aligned}
$$

Here, we note that

$$
\begin{aligned}
\int_{-\infty}^{\infty} \frac{e^{-a^{2} k^{2}}}{k+i(b+K)} d k \\
\quad=-2 i(b+K) \int_{0}^{\infty} \frac{e^{-a^{2} k^{2}}}{k^{2}+(b+K)^{2}} d k \\
\quad=-\frac{\pi i(b+K)}{|b+K|} e^{a^{2}(b+K)^{2}} \operatorname{erfc}(a|b+K|),
\end{aligned}
$$

where

$$
\operatorname{erfc}(x) \equiv \frac{2}{\sqrt{\pi}} \int_{x}^{\infty} e^{-t^{2}} d t
$$

is the complementary error function. Hence,

$$
J=-\pi i e^{a^{2}(b+K)^{2}} \operatorname{erfc}[a(b+K)]
$$

where we have used the relation for $x<0$,

$$
\operatorname{erfc}(x)=2-\operatorname{erfc}(|x|) .
$$

We also note that for a large positive $x$

$$
\operatorname{erfc}(x) \sim \frac{e^{-x^{2}}}{\sqrt{\pi} x}
$$

${ }^{1}$ V. Narayanamurti, Science 213, 717 (1981).

${ }^{2}$ S. Mizuno and S. Tamura, in Proceedings of the 7th International Conference on Phonon Scattering in Condensed Matter, edited by M. Meissner and R. O. Pohl (Springer, Berlin, 1993), p. 471.

${ }^{3}$ S. Mizuno and S. Tamura, Phys. Rev. B 45, 734 (1992).

${ }^{4}$ S. Mizuno and S. Tamura, Phys. Rev. B 45, 13423 (1992).

${ }^{5}$ L. L. Chang, L. Esaki, and R. Tsu, Appl. Phys. Lett. 24, 593 (1974).

${ }^{6}$ F. Capasso, K. Mohammed, and A. Y. Cho, IEEE J. Quantum Electron. QE-22, 1853 (1986).

${ }^{7}$ E. H. Hauge, J. P. Falck, and T. A. Fjeldly, Phys. Rev. B 36, 4203 (1987).

${ }^{8}$ E. H. Hauge and J. A. St $\phi$ vneng, Rev. Mod. Phys. 61, 917 (1989).

${ }^{9}$ S. Mizuno, M. Ito, and S. Tamura, Jpn. J. Appl. Phys. 33, 2880 (1994).
${ }^{10}$ The expressions of $S(N)$ and $C(N)$ for the phonons inside the frequency band are given in Eqs. (15)-(17) of Ref. 4.

${ }^{11}$ E. P. Wigner, Phys. Rev. 98, 145 (1955).

${ }^{12}$ E. Merzbacher, Quantum Mechanics (Wiley, New York, 1970).

${ }^{13}$ D. Bohm, Quantum Theory (Prentice-Hall, New York, 1951).

${ }^{14}$ The explicit expressions of $t$ and $r$ are not given in the previous papers, but they are readily obtained by combining Eqs. (44) and (45) in Ref. 3, and Eqs. (19)-(23) in Ref. 4.

${ }^{15}$ F. Ancilotto, A. Selloni, and E. Tosatti, Phys. Rev. B 40, 3729 (1989).

${ }^{16}$ See, for example, H. J. Maris, C. Thomsen, and J. Tauc, in Phonon Scattering in Condensed Matter, edited by A. C. Anderson and J. P. Wolfe (Springer, Berlin, 1986), p. 374.

${ }^{17}$ A. M. Steinberg, P. G. Kwiat, and R. Y. Chiao, Phys. Rev. Lett. 71, 708 (1993). 